Provided for non-commercial research and education use. Not for reproduction, distribution or commercial use.

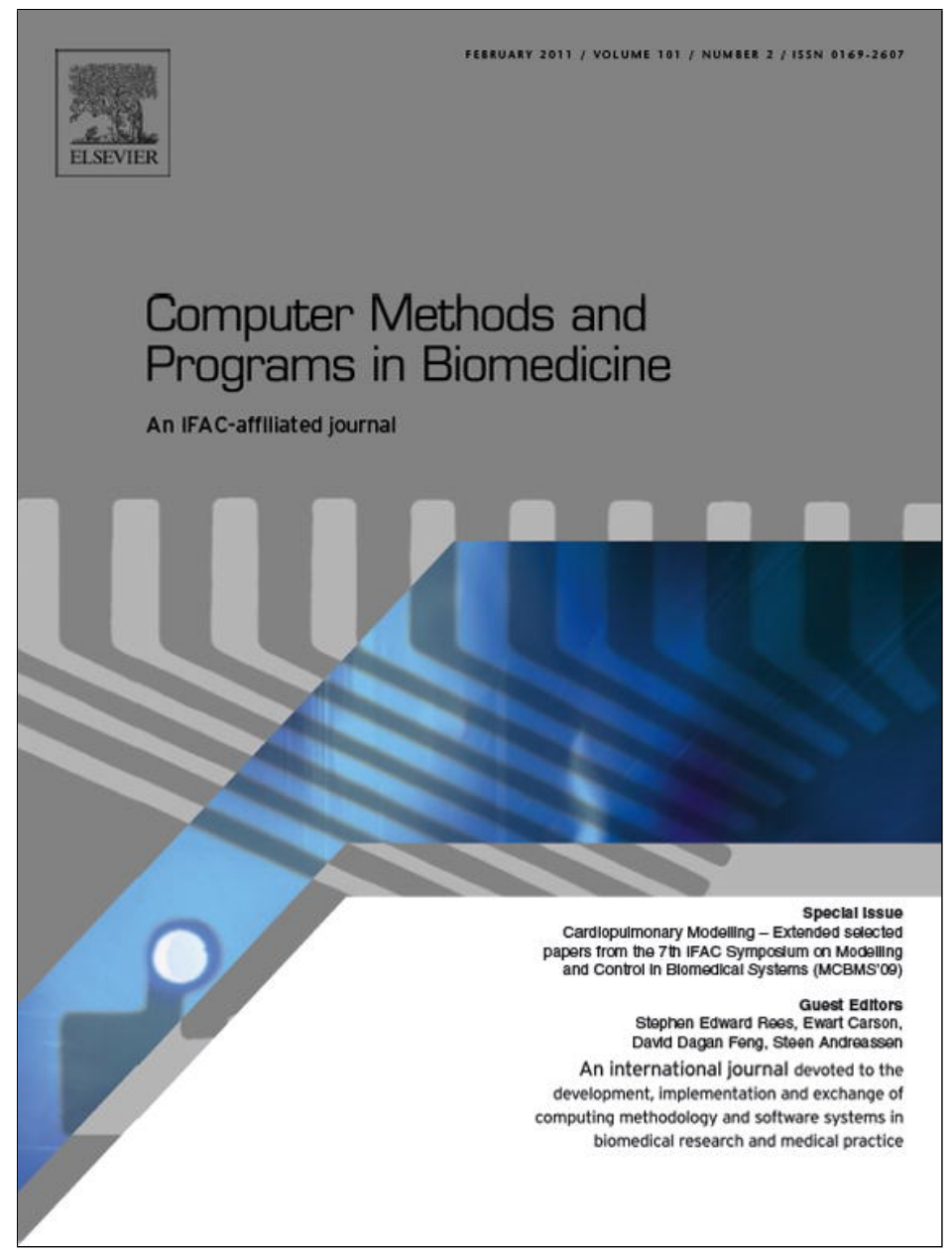

This article appeared in a journal published by Elsevier. The attached copy is furnished to the author for internal non-commercial research and education use, including for instruction at the authors institution and sharing with colleagues.

Other uses, including reproduction and distribution, or selling or licensing copies, or posting to personal, institutional or third party websites are prohibited.

In most cases authors are permitted to post their version of the article (e.g. in Word or Tex form) to their personal website or institutional repository. Authors requiring further information regarding Elsevier's archiving and manuscript policies are encouraged to visit:

http://www.elsevier.com/copyright 


\title{
A 5-component mathematical model for salt-induced hypertension in Dahl-S and Dahl-R rats
}

\author{
Violeta McLoone ${ }^{a, *}$, John Ringwood ${ }^{a}$, Bruce Van Vliet $^{b}$ \\ a Dept. of Electronic Engineering, National University of Ireland Maynooth, Maynooth, Co. Kildare, Ireland \\ b Biomedical Sciences Division, Memorial University, St. John's, Newfoundland, Canada
}

\section{A R T I C L E I N F O}

Article history:

Received 2 December 2009

Received in revised form

3 March 2010

Accepted 22 April 2010

\section{Keywords:}

Hypertension

Mathematical model

Salt intake

Multiple components

Dahl-S

Dahl-R

\begin{abstract}
A B S T R A C T
Salt-induced hypertension has been demonstrated in a variety of species including rats, monkeys, chimpanzees and humans. Until recently, the multiple phases of this blood pressure increase due to high salt intake had not been closely studied. This work builds upon a recent study, which developed a grey-box multi-component model of salt-induced hypertension in the Dahl-S rat. The previous 3-component model has been extended here to include additional model dynamics to improve the model fit and add new important elements to the model response. The model was optimised using numerical techniques with experimental data from 4 different protocols with Dahl-S, Dahl-R and FF2 hybrid rats. Results show a marked improvement over the previous model and confirm the merit of the 5-component model structure. A comparison between the model dynamics for different rat strains has also been included.
\end{abstract}

@ 2010 Elsevier Ireland Ltd. All rights reserved.

\section{Introduction}

Salt-induced hypertension (SIH) is a process whereby a subject develops high blood pressure (BP) due to high salt intake and individuals can be categorised into salt-sensitive or salt-resistant [1,2]. Salt intake has often been linked to the development of hypertension [3-5] and the BP response to salt has been studied in chimpanzees [6], spider monkeys [7], rats [8,9], mice [10] and humans [11].

Until recently, salt-induced hypertension was viewed as a single event and different time courses of the development of hypertension were not distinguished. This was mainly due to a lack of long term data sampled at short enough intervals to determine different phases of the response. Van Vliet et al. [12] first emphasised the presence of two distinct phases of $\mathrm{SIH}$ in Dahl salt-sensitive (Dahl-S) rats and, in addition, their study showed that similar effects were also present to various extents in hybrid rats.

Based on this idea, and using the same experimental data, a multi-component grey-box model was created to simulate the acute and progressive time-courses in BP increase due to high salt intake [13]. The model comprises three dynamic components, as shown in Fig. 1, and was optimised for both Dahl salt-sensitive and hybrid rats. The model was shown to fit the data reasonably well and possible physiological explanations were assigned to each component. A major drawback, however, is the inability of this 3-component model to account for certain features present in the experimental data.

In the present study, the existing 3-component model is extended to improve the data fit and assign a model structure that is possibly more representative of the underlying physiological system. Two new model components were developed using the average data for each experimental protocol (i.e. the

\footnotetext{
* Corresponding author. Tel.: +353879886557.

E-mail address: vmcloone@eeng.nuim.ie (V. McLoone). 0169-2607/\$ - see front matter @ 2010 Elsevier Ireland Ltd. All rights reserved. doi:10.1016/j.cmpb.2010.04.008
} 


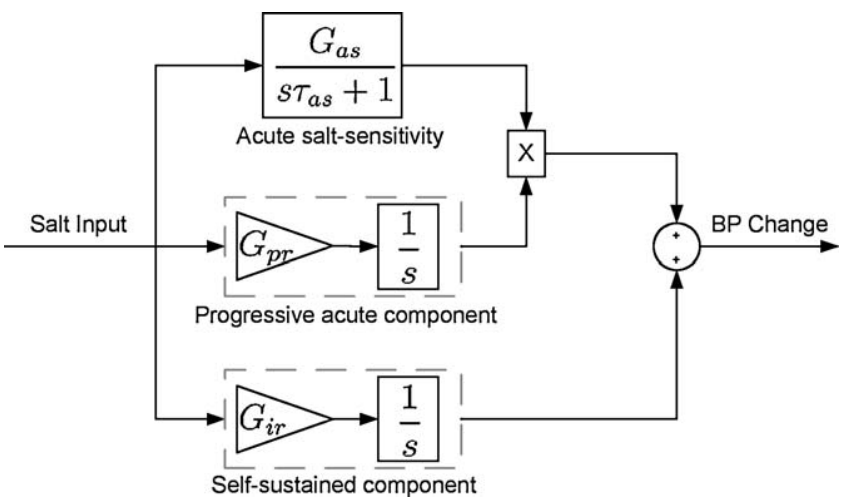

Fig. 1 - 3-Component model structure developed in [13]

'mean animal'), as it is relatively noise-free data and emphasises features present in most if not all animals within a data set. Subsequently, the models were parameterised for each individual animal, including control Dahl salt-resistant (DahlR) animals. Chronic pressure-natriuresis relationships were also plotted for various animals to demonstrate the different features in the progression of SIH.

The remainder of the paper is organised as follows: Section 2 gives a brief overview of the data sets used in this study and Section 3 shows the model structure development. Section 4 describes the numerical techniques employed for model parameter optimisation, while Sections 5 and 6 present the results and discussion of the work.

\section{Experimental data}

Data from four different experimental protocols were used in this study. Plots of the 'mean animal' data for each protocol as well as the respective salt inputs are shown in Figs. 2 and 3.

\subsection{Protocol 1}

Experimental protocol 1, described in detail in [12], was conducted on nine 3-month-old male Dahl-S rats from the Brookhaven strain. A step increase in dietary salt intake was applied for 6 weeks (4\% $\mathrm{NaCl})$, followed by a 4-week recovery period with normal salt intake $(0.7 \% \mathrm{NaCl})$. Blood pressure was recorded using telemeters at a rate of a sample per minute. The 24-h average mean arterial pressure (MAP) was used in the study. A separate set of seven Dahl-R rats were used as control subjects and were fed the same diet as the Dahl-S rats. Fig. 2 shows a plot of the 'mean animal' for the Dahl-S and Dahl-R rats and the associated salt intake.

\subsection{Protocol 2}

Protocol 2 was designed to investigate the reversibility of saltinduced hypertension and was reported in [12]. The protocol included variations in salt intake during a course of eleven weeks. The subjects were five male Dahl-S rats, instrumented with telemeters for blood pressure recording. Weeks 1, 3, 7 and 11 included normal salt diet of $0.7 \% \mathrm{NaCl}$, while during weeks 2, 4-6 and 8-10 the animals were given high salt diet of $4 \%$
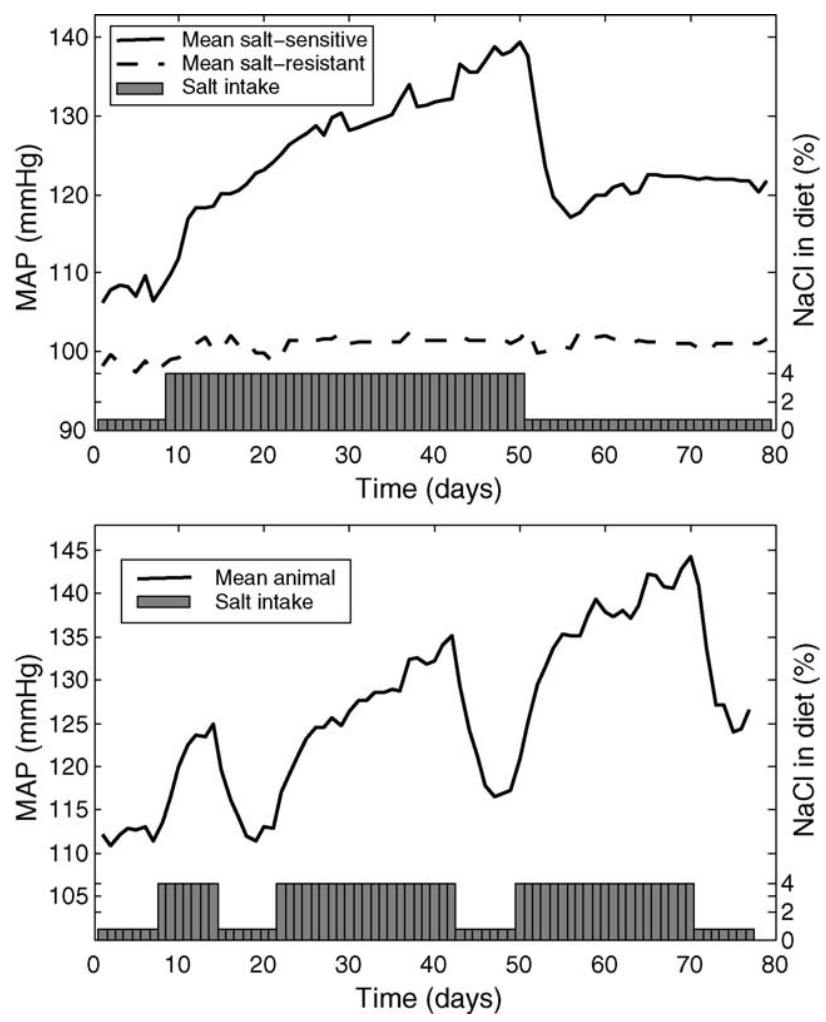

Fig. 2 - 'Mean salt-sensitive' and 'mean salt-resistant' animals of Protocol 1 (top) and 'mean salt-sensitive' animal of Protocol 2 (bottom) and associate salt intake levels.

$\mathrm{NaCl}$. Fig. 2 shows a plot of the 'mean animal' and salt intake for this protocol.

\subsection{Protocol 3}

Protocol 3, described in [13], investigates the effect of shortterm changes in salt intake on the daily MAP level of eight male Dahl-S rats and five control male Dahl-R rats . The salt levels in the diet were varied in a pseudo-random binary sequence where, at each day of the experiment, the level of salt was either changed or it stayed the same. BP telemeters were implanted in all rats and a recovery period was allowed before a high salt diet was given. The protocol was 72 days long and the daily mean BP level was calculated as the average level of MAP sampled once each minute. The dietary salt level was manipulated in the following manner, 00000000011111101010 1100110111011010010011100010111100101000110000100000 , with each ' 0 ' representing a $24 \mathrm{~h}$ exposure to a regular salt diet $(0.7 \% \mathrm{NaCl})$ and each ' 1 ' indicating a $24 \mathrm{~h}$ exposure to high salt diet ( $4 \% \mathrm{NaCl})$. Fig. 3 shows a plot of the 'mean animal' for the salt-sensitive and salt-resistant rats, in addition to the salt intake levels.

\subsection{Protocol 4}

Protocol 4, described in detail in [12] and similarly to Protocol 1 , was conducted to study the BP response to a step increase in salt level in the diet of rats. Thirteen male FF2-hybrid rats (progeny of the cross between Dahl-S and Dahl-R rats) were 

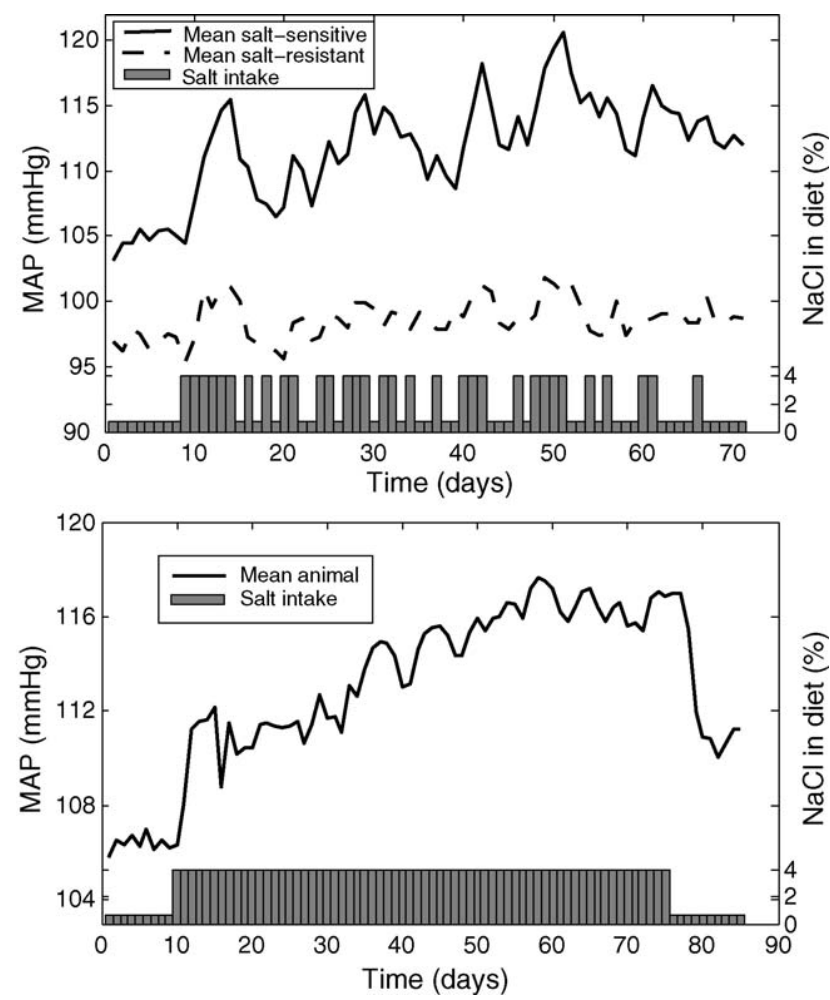

Fig. 3 - 'Mean salt-sensitive' and 'mean salt-resistant' animals of Protocol 3 (top) and 'mean hybrid' animal of Protocol 4 (bottom) and associate salt intake levels.

studied. Following a control week on a regular salt diet containing $0.7 \% \mathrm{NaCl}$, a high salt diet of $4 \% \mathrm{NaCl}$ was given for 10 weeks and then returned back to a regular salt diet for 1 week. BP was measured using implanted telemeters. Fig. 3 shows a plot of the 'mean animal' of the hybrid data set.

\section{Model development}

The existing 3-component model in [13] has salt levels in the diet as input and BP variation from baseline as output, and consists of the following dynamic components:

- An 'acute salt-sensitivity' component, comprising a first order transfer function with steady state gain $G_{a s}$ (mmHg/\% $\% \mathrm{NaCl})$ and time constant $\tau_{\text {as }}$ (days). This component represents the reversible change of BP over a few days to a week, shown in studies in mice [10], humans [14], dogs [15] and monkeys [16].

- A 'progressive self-sustaining' component consisting of a simple integrator with gain $G_{i r}$, simulating a slow, progressive and irreversible BP increase [9,12,17].

- A 'progressive-acute' component consisting of an integrator term with gain $G_{p r}$, which creates a progressive amplification of the gain $G_{a s}$ of the 'acute salt-sensitivity' component of the model. This effect is consistent with progressive worsening of the acute salt-sensitivity and has been demonstrated in chimpanzees [6], rats [18] and humans [19,20].

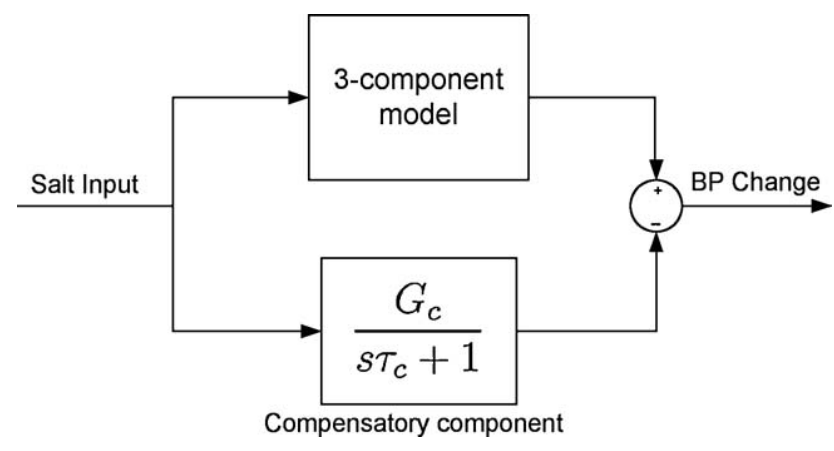

Fig. 4 - 4-Component model structure.

\subsection{4-Component model}

As some features of the data were not well represented by the 3-component model in [13], expansion of the model was required. In Fig. 2, which shows a plot of the 'mean saltsensitive' animal from Protocol 1, after the high salt stimulus is removed $\mathrm{BP}$ recedes to lower levels, undershooting before settling to its new steady-state value. It is impossible to achieve the undershooting response without the addition of an extra component to the existing model.

The new fourth 'compensatory' component, designed to model the overshoot in the data, is shown in Fig. 4. It consists of a first order dynamic component, whose response opposes the increase in BP due to high salt intake. This is generally consistent with the physiology of the blood pressure control system, which is a complex mixture of balancing neural, hormonal and autoregulatory interactions, and thus it is expected that certain mechanisms will counteract the effect of salt on $\mathrm{BP}$, e.g suppression of angiotensin and aldosterone production [21].

\subsection{5-Component model}

A further set of dynamic components were subsequently added to enhance the 4-component model response by accounting for different rates of BP increase and decrease during the stimulation and relaxation phases of the experiments (increase and decrease of salt contents in the diet). This new model component consists of a switch, along with separate 'stimulation' and 'relaxation' dynamics (Fig. 5). These dynamics work in conjunction with the 'acute salt-sensitivity'

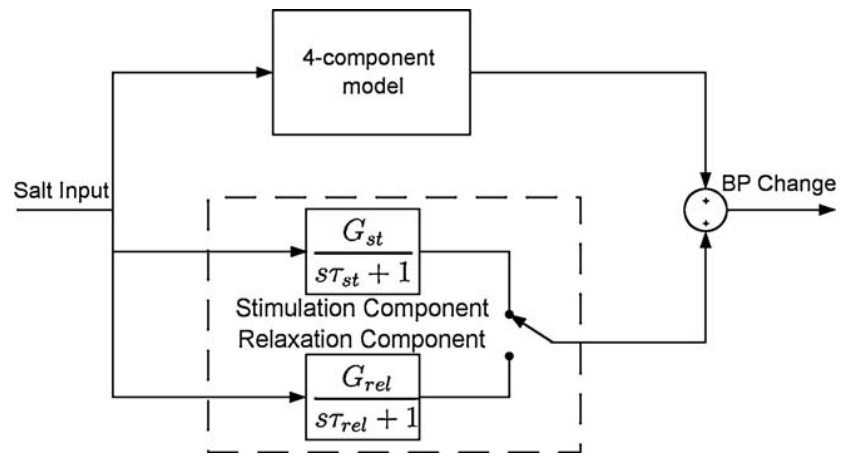

Fig. 5 - 5-Component model structure. 
component, where during high salt intake, the 'stimulation' component is activated, while when the high salt stimulus is removed, the 'relaxation' component takes over. This is reasonable, since substances involved in BP control may have different formation and dispersion mechanisms and associated activation rates.

\subsection{Neural network model}

Neural networks are black-box models, with potentially unlimited level of complexity and capabilities to model nonlinear characteristics. For comparison purposes, a neural network model was implemented in order to assess whether a purely black-box model could perform better for the existing data sets than the 4- and 5- component models of Sections 3.1 and 3.2. This would give an estimate of the degree to which the grey-box model assimilates all the dynamics and characteristics contained in the data.

In order to make the models comparable, the output of the neural network is a multi-step ahead prediction. The network was trained separately for each individual animal. As the data sets are very short and all samples were used during training, no validation data was utilised and thus training was stopped after no more than thirty iterations to avoid potential overfitting. A small number of neurons were employed to enhance parsimony.

Networks with both one and two hidden layers were considered, even though a single layer network can approximate any continuous function [22]. Three single layer and one twolayer networks are presented, with structures $N_{i}-N_{h}-N_{0}$, where $N_{i}$ is the number of input neurons, $N_{h}$ the number of hidden neurons in each layer and $N_{0}$ the number of output neurons. All three single layer structures had $N_{0}=1$, with the current BP value as output of the network, and number of input neurons as follows:

- NNET1: $N_{i}=3$, with inputs consisting of the present value of the salt intake and two past values of the BP output $\left(u_{k}, y_{k-1}, y_{k-2}\right)$,

- NNET2: $N_{i}=4$, with inputs consisting of the present value of the salt intake and three past values of the BP output $\left(u_{k}, y_{k-1}, y_{k-2}, y_{k-3}\right)$, and

- NNET3: $N_{i}=4$, with inputs consisting of the present and one past value of the salt intake and two past values of the BP output $\left(u_{k}, u_{k-1}, y_{k-1}, y_{k-2}\right)$.

A two-layered neural network (NNET4) had $N_{i}=3$, with the inputs of the network including the present value of the salt intake and two past values of the BP output $\left(u_{k}, y_{k-1}, y_{k-2}\right)$. All output values were those predicted by the network. As example, Fig. 6 shows a diagram of NNET1/NNET4.

Different number of hidden layer neurons gave optimal results for the various animals. On average, for all data sets, the number of neurons in the hidden layer, $N_{h}$, was approximately 5.5, with some networks having up to ten hidden layer neurons, while others only three. The simplicity of the 5component model suggests that a low-order structure should be sufficient to represent the main features of the data, and the limited number of training points also restricts the size of the networks.

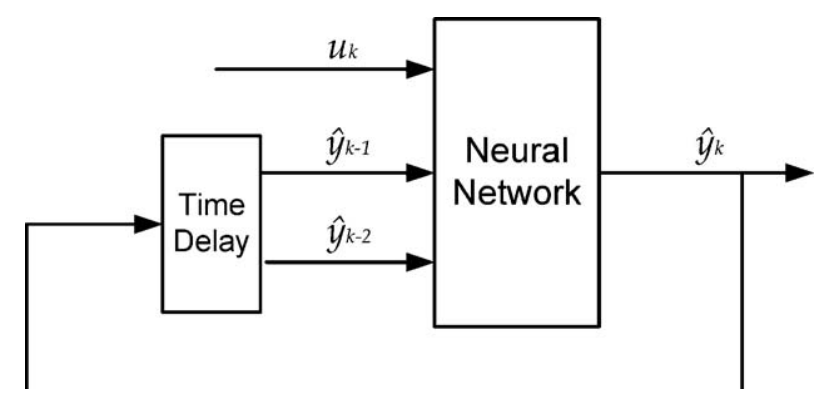

Fig. 6 - Example neural network structure, with $u_{k}$ and $\hat{y}_{k}$ being the current salt input and MAP output of the network respectively, and $\hat{y}_{k-1}$ and $\hat{y}_{k-2}$ being the delay input line formed by two past estimated MAP outputs of the network.

Additional network structures, not presented here, were also considered, including such with additional number of past values of the input and output and various number of hidden neurons; however, increasing the number of connections and weights led to a deterioration of model fit and an increase in errors.

\section{Model optimisation}

All models were parameterised for each individual animal in all four protocols. MatLab's Simulink was used for model implementation, while the parameter optimisation for the 4- and 5-component grey-box models was done using Nelder-Mead simplex algorithm [23], which is suitable for multidimensional unconstrained problems. The objective function to be minimised (J) was defined as the mean squared error (MSE) between model results and experimental data:

$J=\frac{1}{N} \sum_{i=1}^{N}\left(y_{i}-\hat{y}_{i}\right)^{2}$

where $N$ is the number of samples in the data set, $\hat{y}_{i}$ is the blood pressure data obtained from the model simulations, and $y_{i}$ is the experimental blood pressure data.

The black-box neural network models were implemented using MatLab's Neural Network Toolbox. The nonlinear functions in the hidden layer were log sigmoid, while those in the output layer were linear. The Levenberg-Marquardt algorithm [24] was used for network training. The objective function to be minimised was the MSE shown in Eq. (1).

\section{Results}

The model results for the 4-component, 5-component and neural network models are presented here, with the results of the 3-component model given for reference.

\subsection{4-Component model}

Table 1 lists the mean parameter values for the 4-component model in Fig. 4 and their standard deviations for the saltsensitive animals in Protocols 1, 2 and 3. Table 2 shows the 


\begin{tabular}{|c|c|c|c|}
\hline Protocol & Protocol 1 & Protocol 2 & Protocol 3 \\
\hline$G_{a s}$ & $5.792 \pm 2.845$ & $3.819 \pm 2.164$ & $3.076 \pm 2.527$ \\
\hline$\tau_{a s}$ & $2.645 \pm 1.229$ & $2.610 \pm 1.038$ & $2.739 \pm 1.460$ \\
\hline$G_{p r}$ & $0.005 \pm 0.004$ & $0.009 \pm 0.006$ & $0.017 \pm 0.017$ \\
\hline$G_{\text {ir }}$ & $0.130 \pm 0.046$ & $0.138 \pm 0.153$ & $0.074 \pm 0.027$ \\
\hline$G_{c}$ & $4.584 \pm 3.199$ & $8.540 \pm 10.684$ & $4.732 \pm 3.872$ \\
\hline$\tau_{c}$ & $18.609 \pm 10.898$ & $30.659 \pm 24.978$ & $19.324 \pm 12.249$ \\
\hline
\end{tabular}

Table 2 - Mean parameters and standard deviations for the 4-component model for the control Dahl-R rats of Protocols 1 and 3 and hybrid rats of Protocol 4.

\begin{tabular}{llll} 
Protocol & \multicolumn{1}{c}{ Protocol 1 } & \multicolumn{1}{c}{ Protocol 3 } & Protocol 4 \\
\hline$G_{\text {as }}$ & $0.959 \pm 0.541$ & $2.256 \pm 0.482$ & $2.851 \pm 1.558$ \\
$\tau_{a s}$ & $1.585 \pm 0.237$ & $2.194 \pm 0.204$ & $3.029 \pm 0.844$ \\
$G_{p r}$ & $0.0005 \pm 0.001$ & $0.0027 \pm 0.002$ & $0.002 \pm 0.002$ \\
$G_{\text {ir }}$ & $0.038 \pm 0.021$ & $0.017 \pm 0.019$ & $0.031 \pm 0.020$ \\
$G_{c}$ & $1.434 \pm 1.833$ & $2.537 \pm 0.549$ & $1.989 \pm 1.654$ \\
$\tau_{c}$ & $24.932 \pm 20.368$ & $5.558 \pm 1.338$ & $7.368 \pm 3.739$ \\
\hline
\end{tabular}

Table 3 - Mean parameters and standard deviations for the 5-component model for the Dahl-S rats of Protocols 1,2 and 3.

\begin{tabular}{llll} 
Protocol & Protocol 1 & Protocol 2 & \multicolumn{1}{c}{ Protocol 3 } \\
\hline$G_{\text {as }}$ & $4.785 \pm 1.329$ & $2.748 \pm 1.057$ & $4.017 \pm 1.848$ \\
$\tau_{\text {as }}$ & $3.056 \pm 3.959$ & $1.884 \pm 0.972$ & $2.186 \pm 0.639$ \\
$G_{p r}$ & $0.001 \pm 0.001$ & $0.011 \pm 0.012$ & $0.001 \pm 0.001$ \\
$G_{i r}$ & $0.122 \pm 0.032$ & $0.097 \pm 0.119$ & $0.072 \pm 0.055$ \\
$G_{c}$ & $5.029 \pm 2.881$ & $3.903 \pm 3.471$ & $4.356 \pm 1.836$ \\
$\tau_{c}$ & $9.103 \pm 5.881$ & $13.326 \pm 10.873$ & $7.047 \pm 1.969$ \\
$G_{\text {st }}$ & $4.178 \pm 3.055$ & $2.692 \pm 1.787$ & $3.121 \pm 1.195$ \\
$\tau_{\text {st }}$ & $9.246 \pm 4.389$ & $9.640 \pm 6.263$ & $19.201 \pm 8.338$ \\
$G_{\text {rel }}$ & $3.871 \pm 1.689$ & $1.468 \pm 2.112$ & $0.973 \pm 1.557$ \\
$\tau_{\text {rel }}$ & $3.468 \pm 5.253$ & $3.321 \pm 5.915$ & $0.635 \pm 0.315$ \\
\hline
\end{tabular}

mean parameter values for the control salt-resistant animals of Protocols 1 and 3 and the hybrid animals of Protocol 4. The 'acute salt-sensitivity' gain $G_{a s}$ is clearly lowest for the salt-resistant animals from Protocols 1 and 3. In addition, the mean $G_{a s}$ value of the hybrid animals is higher than that of the salt-resistant rats, but lower than those of the salt-sensitive animals. This is very reasonable since hybrid animals have a selection of genetic types, varying from salt-sensitive to saltresistant. The time constant $\tau_{a s}$ is similar across all protocols and varies between 1.5 and 3 days, which is consistent with other rat studies [25]. The 'self-sustained' component gain $G_{i r}$ is also lowest for the salt-resistant and hybrid rats, and highest for the salt-sensitive animals of Protocol 1, exposed to prolonged, uninterrupted high salt intake.

\subsection{5-Component model}

Table 3 shows the mean parameter values and standard deviations (SD) for the salt-sensitive animals of Protocols 1, 2 and 3 for the 5-component model, while Table 4 presents the parameter values and SDs for the control salt-resistant animals of Protocol 1 and 3 and hybrid animals of Protocol 4. As before, the gains of the salt-sensitive animals are consistently higher than those of the salt-resistant and hybrid rats. The mean $G_{a s}$
Table 4 - Mean parameters and standard deviations for the 5-component model for the control Dahl-R rats of Protocols 1 and 3 and the hybrid rats of Protocol 4.

\begin{tabular}{llll} 
Protocol & \multicolumn{1}{c}{ Protocol 1 } & \multicolumn{1}{c}{ Protocol 3 } & \multicolumn{1}{c}{ Protocol 4 } \\
\hline$G_{\text {as }}$ & $1.759 \pm 1.388$ & $2.806 \pm 0.892$ & $1.841 \pm 0.662$ \\
$\tau_{\text {as }}$ & $0.752 \pm 0.246$ & $1.999 \pm 0.596$ & $1.587 \pm 0.836$ \\
$G_{p r}$ & $0.0006 \pm 0.0004$ & $0.0008 \pm 0.0007$ & $0.001 \pm 0.002$ \\
$G_{i r}$ & $0.014 \pm 0.013$ & $0.024 \pm 0.018$ & $0.027 \pm 0.021$ \\
$G_{c}$ & $2.007 \pm 0.887$ & $3.256 \pm 0.942$ & $1.653 \pm 1.229$ \\
$\tau_{c}$ & $4.114 \pm 1.518$ & $4.746 \pm 1.0332$ & $5.552 \pm 2.438$ \\
$G_{s t}$ & $1.014 \pm 0.421$ & $0.457 \pm 0.3193$ & $1.274 \pm 1.065$ \\
$\tau_{\text {st }}$ & $4.142 \pm 1.460$ & $12.795 \pm 6.718$ & $11.257 \pm 7.432$ \\
$G_{r e l}$ & $2.338 \pm 1.444$ & $0.048 \pm 0.089$ & $2.177 \pm 1.703$ \\
$\tau_{\text {rel }}$ & $40.48 \pm 50.864$ & $0.526 \pm 0.221$ & $1.785 \pm 2.369$ \\
\hline
\end{tabular}

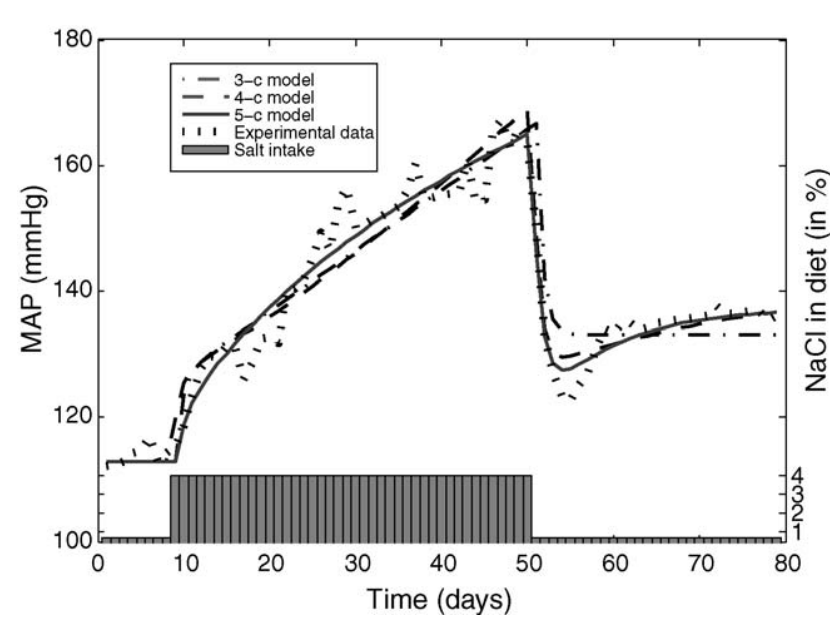

Fig. 7 - Model response for all three grey-box models (3-component, 4-component and 5-component models) compared to the experimental data for salt-sensitive animal SB11 from Protocol 1.

for the Dahl-S rats of Protocol 1 is an exception, with the value being quite similar to that of the salt-resistant and hybrid animals. However the acute reversible response in this model is a combination of the 'acute salt-sensitivity' and 'stimulation' dynamics and $G_{\text {st }}$ compensates in this case by being much larger for the salt-sensitive animals.

The advantages of employing different dynamic components for the stimulation and relaxation stages of the experiment become clear with the 5-component model. For the salt-sensitive, and even for the hybrid, animals in Tables 3 and 4, the time constant values of the stimulation dynamics $\tau_{\text {st }}$ are prominently larger than those of the relaxation component $\tau_{\text {rel }}$ for all protocols. This helps improve the model fit, compared to the 3-component and 4-component models, as shown in Fig. 7, where the responses of the different models are compared for one particular animal (SB11). The time constant values are more unreliable in the case of the salt-resistant animals, as the lack of prominent response to salt intake in these animals may not necessitate all available model components.

A comparison of the mean MSEs and SDs for each protocol is shown in Table 5 for the salt-sensitive animals. Table 6 shows the same set of results for the control salt-resistant and hybrid animals. Each additional component included in 
Table 5 - Mean MSEs and standard deviations for each model for the salt-sensitive animals of Protocols 1, 2 and 3.

\begin{tabular}{cccc} 
Protocol & 3-Comp & 4-Comp & 5-Comp \\
\hline Protocol 1 & $6.664 \pm 5.780$ & $5.362 \pm 3.290$ & $4.066 \pm 1.831$ \\
Protocol 2 & $9.119 \pm 6.126$ & $7.743 \pm 6.036$ & $6.863 \pm 5.439$ \\
Protocol 3 & $6.455 \pm 1.950$ & $4.281 \pm 1.410$ & $3.181 \pm 1.115$ \\
\hline
\end{tabular}

Table 6 - Mean MSEs and standard deviations for the control salt-resistant animals of Protocols 1 and 3 and the hybrid animals of Protocol 4 for each model.

\begin{tabular}{cccc} 
Protocol & 3-Comp & 4-Comp & 5-Comp \\
\hline Protocol 1 & $2.620 \pm 0.911$ & $2.184 \pm 1.000$ & $1.775 \pm 0.779$ \\
Protocol 3 & $2.993 \pm 0.407$ & $2.570 \pm 0.614$ & $2.283 \pm 0.617$ \\
Protocol 4 & $2.935 \pm 3.160$ & $2.573 \pm 2.181$ & $2.261 \pm 1.634$ \\
\hline
\end{tabular}

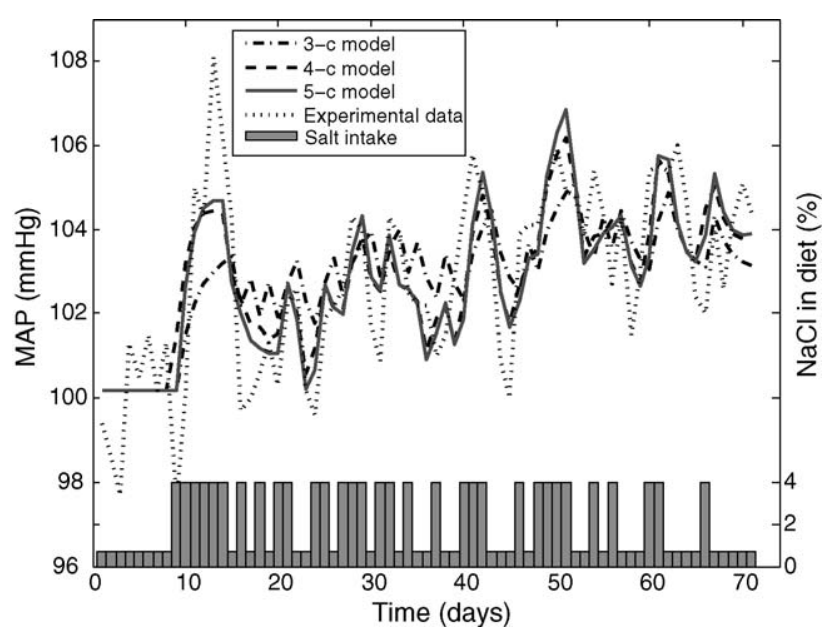

Fig. 8 - Model response for all three grey-box models (3-component, 4-component and 5-component models) compared to the experimental data for salt-resistant animal RB20 from Protocol 3.

the model leads to a marked reduction in MSE for the saltsensitive animals. There is a modest reduction in error for the salt-resistant and hybrid animals. However, it is not significant, since an increase in the number of model components may not be justifiable in the case of salt-resistant animals, as such model components may not be significantly activated in Dahl-R and hybrid rats. Fig. 8 shows a plot of the responses of the various models compared to the experimental data for salt-resistant animal RB20 from Protocol 3. There is no large improvement in the response with increasing model complexity.

An F-test [26] was performed to assess whether the increase in the model complexity is justified for the saltsensitive and hybrid animals. In general, an F-test value is calculated to compare the performances of two models, a simple and a more complex one, with respect to the number of model parameters. As the result of the test is, essentially, a ratio of performances versus complexity between the simpler and the more complex models, an F-test value of 1 signifies that the two models perform equally well for their respective degree of complexity, while an F-test value greater than 1 signifies some merit in the use of the more complex model. The
Table 7 - Mean F-test values for the salt-sensitive animals of Protocols 1-3 and the ybrid animals in

Protocol 4 and associated confidence intervals ( $P$-values). $F 1$ is the $F$-value corresponding to the increase of model

complexity from three to four components, while F2 refers to the $F$-value corresponding to the increase of model complexity from four to five components.

\begin{tabular}{lrccc} 
Protocol & F1 & P-value 1 & F2 & P-value 2 \\
\hline Protocol 1 & 5.016 & 0.01 & 4.443 & 0.005 \\
Protocol 2 & 10.764 & 0.001 & 2.111 & 0.1 \\
Protocol 3 & 15.601 & 0.001 & 5.108 & 0.01 \\
Protocol 4 & 2.122 & $>0.1$ & 1.755 & $>0.1$ \\
\hline
\end{tabular}

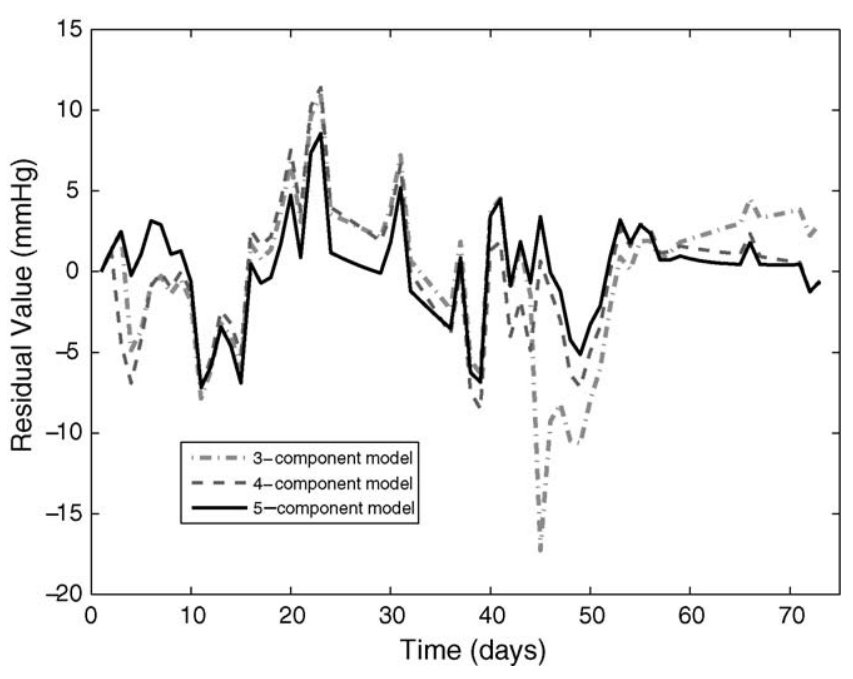

Fig. 9 - Residuals for one animal from Protocol 1 (SB11).

degree to which the F-test value exceeds one can also be used as a measure of confidence interval on the $F$-value, taking into account the number of samples in the data set and the number of parameters used. In our study, two F-test values were calculated for the salt-sensitive and hybrid animals, one comparing the 3- and 4-component models (F1) and the other comparing the 4- and 5-component models (F2). The two mean F-test values with their associated confidence intervals are presented in Table 7 for each protocol. From the results it can be seen that increase in complexity both from 3 to 4 components and from 4 to 5 components is justifiable with very high confidence for all protocols except for the one with hybrid animals (Protocol 4). In the hybrid animal data set, the F-test values are greater than one; however, the confidence intervals are lower than those for Protocols 1-3. Since some of the hybrid animals have genetic make-up similar to that of salt-resistant animals [27], and thus lack some of the response features of the salt-sensitive animals, it appears that not all model components are necessary to represent their responses. It is the intent, however, to have a general model, which can represent both salt-sensitive and salt-resistant animals, thus some model components may inadvertently appear redundant in the latter case but are essential in the former.

The residual between the modelled and actual BP data can also be used as a measure of model quality. A plot of the residuals for one salt-sensitive animal (SB11) from Protocol 1 is shown in Fig. 9, while the autocorrelation of the 


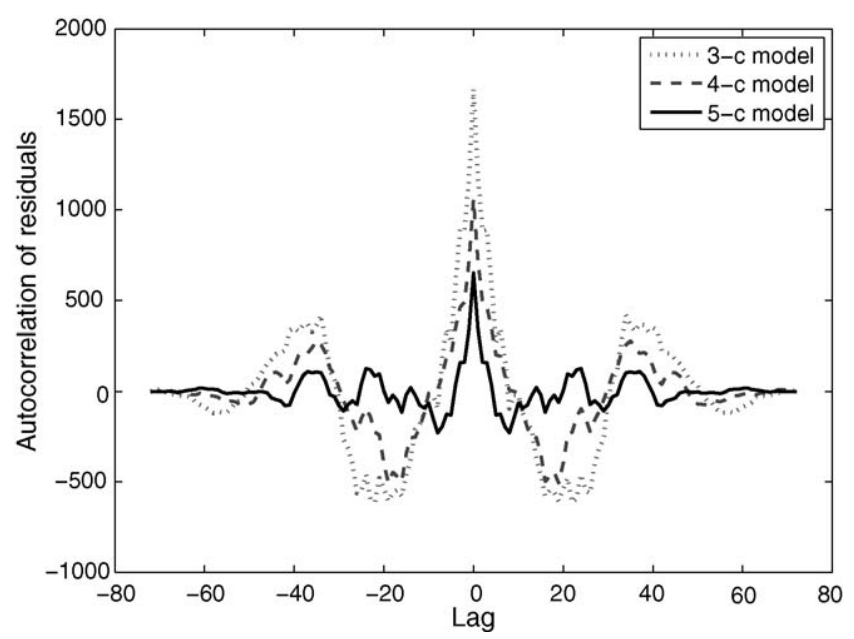

Fig. 10 - Autocorrelation of residuals for one animal from Protocol 1 (SB11).

residuals is shown in Fig. 10. Both plots confirm improvement with the addition of component 4 and then component 5 to the model. With each new component the residuals become less correlated, thus approaching white noise, confirming that the majority of the signal is explained by the 5 -component model and the addition of any further component would not be required, as the small improvement in fit would be unlikely to justify any further increase in complexity.

Figs. 11 and 12 show plots of the 5-component model results for the 'mean animal' for each protocol and compare them to the experimental data. These plots confirm the quality of the model fit and give merit to the model structure.

\subsection{Neural networks}

Table 8 shows the mean MSEs and standard deviations for the various neural network models for the salt-sensitive and hybrid animals. Surprisingly, results are not as good as those for the grey-box models. There are a number of potential reasons for the poor response of the neural networks. The data sets have very few samples $(j 80)$ and the networks were trained on individual animals only. No validation data was used, with less than 80 data points, therefore training had to be stopped early to avoid over-fitting. With further refinement of the neural network models arguably better results could have been obtained. However, the purpose was to assess how well models with strictly defined structure and based on some physiological principles (the 4- and 5-component models) could perform compared to purely black box models.

\section{Discussion}

The 5-component model developed here is, so far, the most comprehensive model of the dynamics of salt-induced hypertension in Dahl-S rats. The model fits the data very well and the components are reasonably intuitive and have some physiological basis. Table 5 shows the large reduction in error in the 5-component model compared to the initial 3component model. In addition, the autocorrelation of the
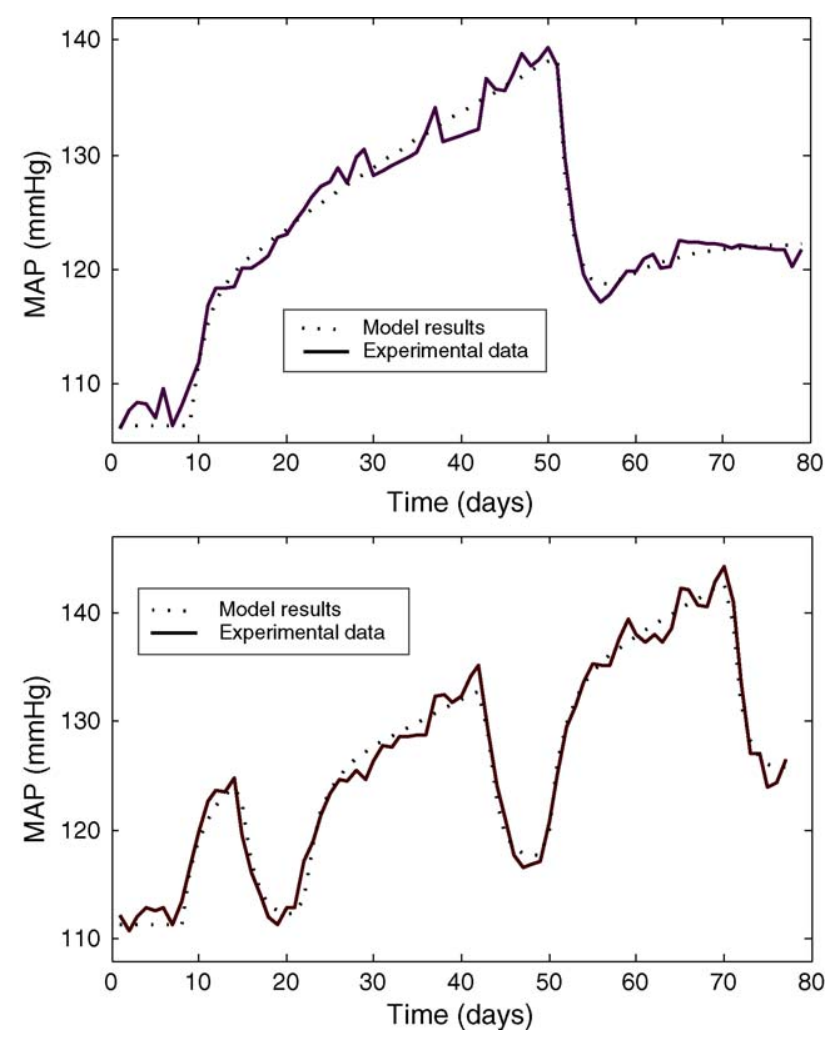

Fig. 11 - 5-Component model response compared to experimental output for the 'mean salt-sensitive animal' of Protocol 1 (top) and Protocol 2 (bottom).

residuals approaches that of white noise, signifying a relatively complete model structure.

Fig. 7 also shows the type of improvement in model response that the additional components (4 and 5) bring to the model over the 3-component model. New elements of the data are featured, in particular the differing time constants between stimulation and relaxation and the undershoot of BP when high-salt intake is ceased.

The neural network models, and their inferior performance compared to the grey-box models, have manifested the importance in using physiological knowledge in model development. Arguably, the neural network models could have been further refined in order to achieve better MSE results. However, it is futile to strive for minimal errors, when no practical structural knowledge can be gained from the system. Moreover, the comparison between the 5-component model and the neural network emphasises again that any further increase in model complexity would not necessarily improve the model response.

In previous studies, the slope of the pressure-natriuresis relationship has been used to define the acute salt sensitivity of BP $[28,29]$. The 5 -component grey-box model developed here can be used to generate pressure-natriuresis relationship curves, which manifest the progression of the development of salt-induced hypertension and worsening of the subjects' salt-sensitivity with prolonged high salt intake.

A combination of the 'acute salt-sensitivity', 'compensatory' and 'stimulation' components impact on the initial 
Table 8 - Mean MSEs and STDs for the neural network models for all protocols.

\begin{tabular}{lccc} 
Protocol & NNET1 & NNET2 & NNET3 \\
\hline Protocol 1 & $11.860 \pm 8.699$ & $16.754 \pm 12.142$ & $12.960 \pm 10.469$ \\
Protocol 2 & $27.788 \pm 23.892$ & $32.044 \pm 23.070$ & $47.550 \pm 45.332$ \\
Protocol 3 & $11.586 \pm 3.335$ & $14.278 \pm 5.783$ & $15.073 \pm 6.528$ \\
Protocol 4 & $5.241 \pm 4.711$ & $6.007 \pm 4.860$ & $4.411 \pm 2.633$ \\
\hline
\end{tabular}
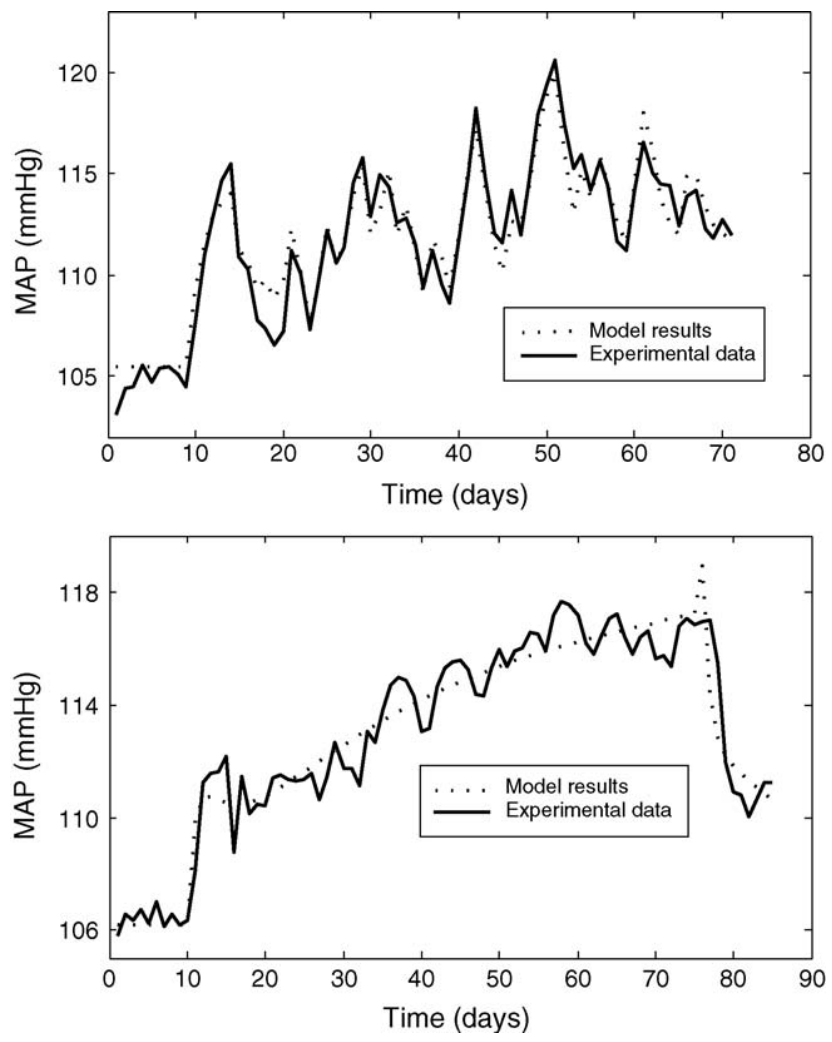

Fig. 12 - 5-Component model response compared to experimental output for the 'mean salt-sensitive animal' of Protocol 3 (top) and the 'mean hybrid animal' from Protocol 4 (bottom).

slope of the chronic pressure-natriuresis relationship. The slope is determined by the magnitude of the animal's response to high salt intake and is inversely proportional to the $G_{\text {as }}$ and $G_{s t}$ gains. Thus, for animals, which are very salt-sensitive the slope is of a low value. The 'progressive acute' component in the model results in a further reduction of the slope value with continued exposure to high salt diet and this reduction is inversely proportional to $G_{p r}$. This effect corresponds to an increase in acute salt-sensitivity with time. The 'selfsustained' or irreversible component in the model contributes to the pressure-natriuresis relationship by shifting the curve to the right, i.e. towards higher resting BP levels [12,17]. Since this component of the BP response is irreversible, the shift in the pressure-natriuresis curve is also permanent and the $G_{\text {ir }}$ gain is proportional to the amount of rightward shift in the curve. All of the effects described here can be clearly seen in Fig. 13 for the salt-sensitive animal SB11 from Protocol 1. In comparison, Fig. 14 shows the pressure-natriuresis relationships for a salt-resistant animal from Protocol 1. The rightward shift in the curve (notice the scale difference) is very small

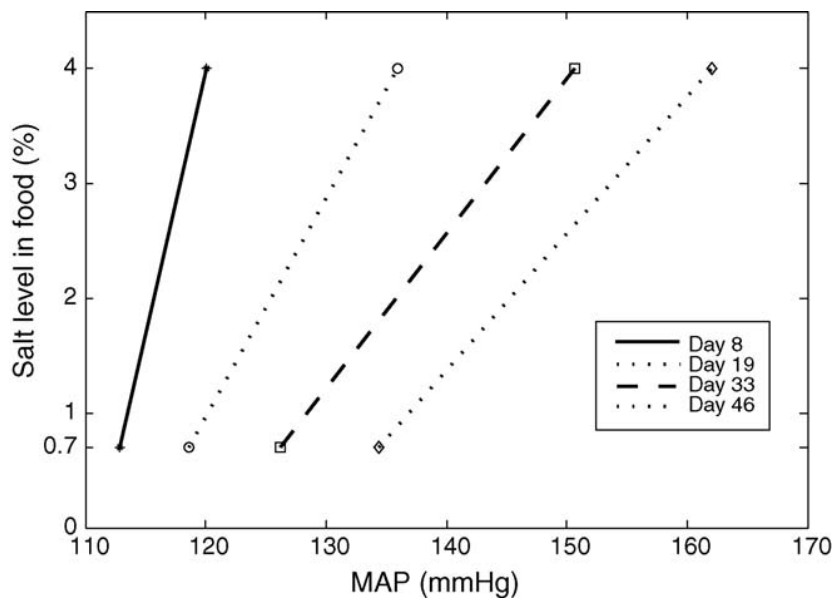

Fig. 13 - Pressure-natriuresis curves for salt-sensitive animal SB11.

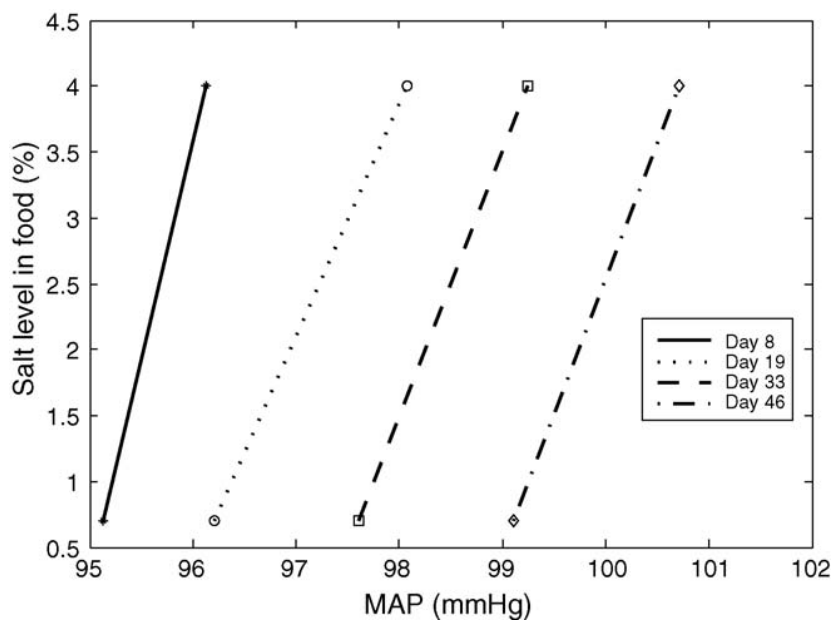

Fig. 14 - Pressure-natriuresis curves for salt-resistant animal RB2.

compared to that of the salt-sensitive animal, and there is no overall reduction in the slope of the relationship. Finally, Fig. 15 shows the pressure-natriuresis curve of hybrid animal FF6 from Protocol 4. In this case, there is no rightward shift in the curve, signifying no irreversible component, and a very small reduction in the slope of the curve. These results demonstrate the ability of the model to distinguish between different mechanisms responsible for the development of salt-induced hypertension and more importantly show the characteristic difference between BP responses of salt-sensitive and saltresistant animals.

It is not within our expertise to assign exact physiological meaning to the various additional dynamics in the model. Nevertheless, it is believed that the 5-component 


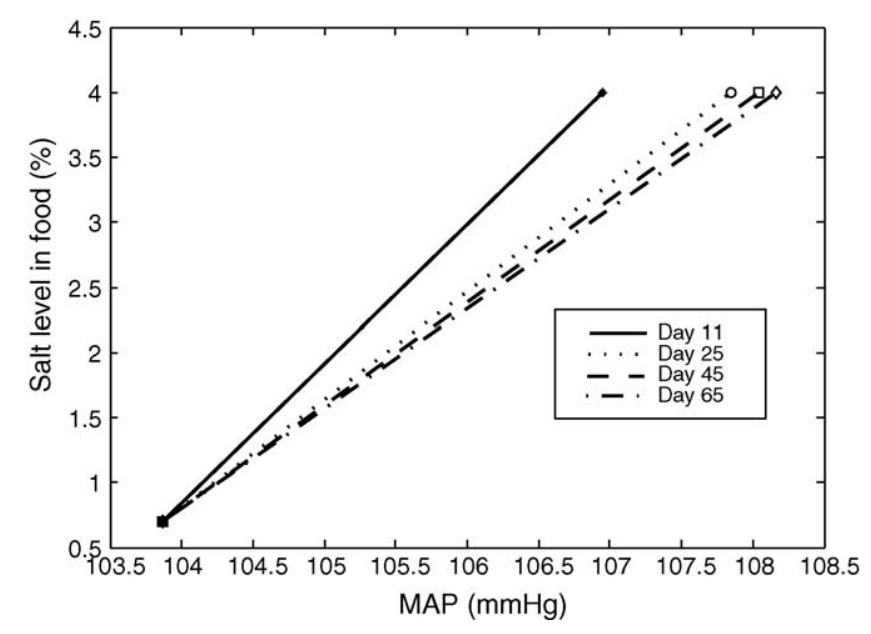

Fig. 15 - Pressure-natriuresis curves for hybrid animal FF6.

model articulates clear and distinct characteristics, which may correspond with separate physiological phenomena. The combination of 'acute salt-sensitivity' and 'progressive acute' components coincides with the time course of reestablishment of salt balance within days. The 'self-sustained' component, leading to an irreversibility of BP increase, could be attributed to the vascular remodelling and/or renal damage such as fibrosis and hypertrophy or lesions [30-33]. The 'compensatory' component could be related to mechanisms such as the suppression of the renin-angiotensin-aldosterone system, which opposes BP increase, while the combination of 'stimulation' and 'relaxation' dynamics, can be attributed to a hysteresis effect, typical of systems where substances are produced and dispersed at different rates.

\section{Conclusions}

This study highlights the multi-component nature of saltinduced hypertension by describing two additional model components to an already existing model [13], which represent the time course of $\mathrm{BP}$ response to high salt intake in Dahl-S and hybrid rats. The previous 3-component model in [13] represented a good starting point in the modelling of saltinduced hypertension but lacked the complexity to depict all major features of the BP response.

The 5-component model has helped gain an insight into the processes involved in blood pressure control and it is important for future experimental protocols to be designed in view of the fact the BP increase due to salt intake has numerous components, some reversible and others irreversible.

We believe that appropriate experiments should be conducted in order to differentiate between the various response components and to attempt to understand their exact physiological significance. In the future, additional excitation signals and protocols may be studied in order to provide the model with the ability to model inter-animal variation in $\mathrm{BP}$, as presently the model is optimised to simulate the response of individual animals only. In addition, experiments similar to the ones described in this work, are currently being carried out on Sprague Dawley rats in Prof. Van Vliet's Laboratory. These may help confirm the suitability of the 5-component model structure to fit data from a wider variety of animals, ranging from normotensive to hypertensive.

\section{Conflict of interest statement}

None declared.

\section{REFERENCES}

[1] T. Kawasaki, C. Delea, F. Bartter, H. Smith, The effect of high-sodium and low-sodium intakes on blood pressure and other related variables in human subjects with idiopathic hypertension, Americal Journal of Medicine 64 (1978) 193-198.

[2] T. Fujita, W. Henry, F. Bartter, C. Lake, C. Delea, Factors influencing blood pressure in salt-sensitive patients with hypertension, American Journal of Medicine 69 (1980) 334-344.

[3] F. He, G. MacGregor, Effect of longer-term modest salt reduction on blood pressure, Cochrane Database of Systematic Reviews (2005), 2:CD004937.

[4] M. Joffres, N. Campbell, B. Manns, K. Tu, Estimate of the benefits of a population-based reduction in dietary sodium additives on hypertension and its related health care costs in Canada, Canadian Journal of Cardiology 23 (2007) 437-443.

[5] P. Meneton, X. Jeunemaitre, H. de Wardener, G. MacGregor, Links between dietary salt intake, renal salt handling, blood pressure, and cardiovascular diseases, Physiological Reviews 85 (2005) 679-715.

[6] D. Denton, R. Weisinger, N. Mundy, E. Wickings, A. Dixons, P. Moisson, A. Pingard, R. Shade, D. Carey, R. Ardaillou, F. Paillard, J. Chapman, J. Thillet, J. Michel, The effect of increased salt intake on blood pressure of chimpanzees, Nature Medicine 1 (10) (1995) 1009-1016.

[7] S. Srinivasan, G. Berenson, B. Radhakrishnamurthy, E. Dalferes, D. Underwood, T. Foster, Effects of dietary sodium and sucrose on the induction of hypertension in spider monkeys, American Journal of Clinical Nutrition 33 (1980) 561-569.

[8] G. Meneely, R. Tucker, W. Darby, S. Auerbach, Chronic sodium chloride toxicity in the albino rat. II. Occurrence of hypertension and of a syndrome of edema and renal failure, Journal of Experimental Medicine 98 (1953) 71-80.

[9] L. Dahl, Effects of chronic excess salt feeding, Journal of Experimental Medicine 114 (1961) 231-236.

[10] A. Leonard, L. Chafe, J.-P. Montani, B. Van Vliet, Increased salt sensitivity in eNOS knockout mice, American Journal of Hypertension 12 (2006) 1264-1269.

[11] K. O'Shaughnessy, F. Karet, Salt handling and hypertension, Annual Revue of Nutrition 26 (2006) 343-365.

[12] B. Van Vliet, L. Chafe, S. Halfyard, A. Leonard, Distinct rapid and slow phases of salt-induced hypertension in dahl salt-sensitive rats, Journal of Hypertension 24 (2006) 1599-1606.

[13] V. McLoone, J. Ringwood, B. Van Vliet, Multi-component mathematical model of salt sensitivity in Dahl-S rats, BMC-Physiology (2009) 9-20.

[14] J. McDonough, C. Wilhelmj, The effect of excess salt intake on human blood pressure, American Journal of Digestive Diseases 21 (1954) 180-181.

[15] T. Coleman, A. Guyton, Hypertension caused by salt loading in the dog. III. Onset transients of cardiac output and other circulatory variables, Circulation Research 25 (1969) 153-160. 
[16] S. Srinivasan, E. Dalferes, R. Wolf, B. Radhakrishnamurthy, T. Foster, G. Berenson, Variability in blood pressure response to dietary sodium intake among African green monkeys (Cercopithecus aethiops), American Journal of Clinical Nutrition 39 (1984) 792-796.

[17] B. Van Vliet, J.-P. Montani, The time course of salt-induced hypertension, and why it matters, International Journal of Obesity 32 (2008) 35-S47.

[18] L. Dahl, K. Knudsen, M. Heine, G. Leitl, Effects of chronic excess salt ingestion. Modification of experimental hypertension in the rat by variations in the diet, Circulation Research 22 (1968) 11-18.

[19] J. Myers, T. Morgan, The effect of sodium intake on the blood pressure related to age and sex, Clinical and Experimental Hypertension 5 (1983) 99-118.

[20] M. Weinberger, N. Fineberg, Sodium and volume sensitivity of blood pressure. Age and pressure change over time, Hypertension 18 (1991) 67-71.

[21] B. Cholewa, D. Mattson, Role of the renin-angiotensin system during alterations of sodium intake in conscious mice, American Journal of Physiology Regulatory Integrative and Comparative Physiology 281 (2001) 987-993.

[22] K. Funahashi, On the approximate realization of continuous mappings by neural networks, Neural Networks 2 (3) (1989) 183-192.

[23] J. Lagarias, J. Reeds, M. Wright, P. Wright, Convergence properties of the Nelder-Mead simplex method in low dimensions, SIAM Journal of Optimization 9 (1) (1998) 112-147.

[24] D.W. Marquardt, An algorithm for the least-squares estimation of nonlinear parameters, SIAM Journal of Applied Mathematics 11 (2) (1963) 431-441.

[25] J. Osborn, B. Hornfeldt, Arterial baroreceptor denervation impairs long-term regulation of arterial pressure during dietary salt loading, American Journal of Physiology 275 (1998) 1558-1566.

[26] T. Ludden, S. Beal, L. Sheiner, Comparison of the Akaike information criterion, the Schwarz criterion and the $F$ test as guides to model selection, Journal of Pharmacokinetics and Pharmacodynamics 22 (5) (1994) 431-445.

[27] K. Knudsen, L. Dahl, K. Thompson, J. Iwai, M. Heine, G. Leitl, Effects of chronic excess salt ingestion. Inheritance of hypertension in the rat, Journal of Experimental Medicine 132 (1970) 976-1000.

[28] A.C. Guyton, Arterial Pressure and Hypertension, WB Saunders and Co., London, 1980.

[29] G. Kimura, B. Brenner, Implications of the linear pressure-natriuresis relationship and importance of sodium sensitivity in hypertension, Journal of Hypertension 15 (1997) 1055-1061.

[30] J. Rapp, H. Dene, Development and characteristics of inbred strains of Dahl salt-sensitive and salt-resistant rats, Hypertension 7 (1985) 340-349.

[31] D. Jaffé, L. Sutherland, D. Barker, L. Dahl, Effects of chronic excess salt ingestion. Morphologic findings in kidneys of rats with differing genetic susceptibilities to hypertension, Archives of Pathology 90 (1970) 1-16.

[32] P. Chen, P. St John, K. Kirk, D. Abrahamson, P. Sanders, Hypertensive nephrosclerosis in the Dahl/Rapp rat. Initial sites of injury and effect of dietary L-arginine supplementation, Laboratory Investigation: A Journal of Technical Methods and Pathology 68 (1993) 174-184.

[33] R. Johnson, J. Herrera-Acosta, G. Schreiner, B. Rodriguez-Iturbe, Subtle acquired renal injury as a mechanism of salt-sensitive hypertension, New England Journal of Medicine 346 (2002) 913-923. 